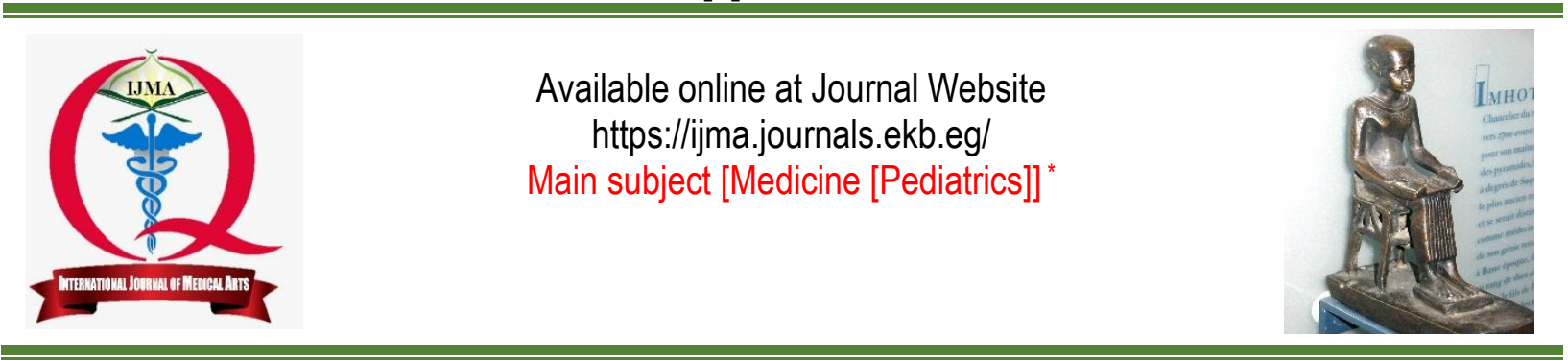

Original article

\title{
Frequency of Mitral Valve Prolapse in Apparently Healthy Children in Alexandria Governorate
}

\author{
Nada Alaa Mohamed Diabª ${ }^{\mathbf{a}}$ Lotfy Abdel-Fattah El-Seheimy ${ }^{\mathbf{b}}$; Aly Mohamed Abd Elmohsen ${ }^{\mathbf{c}}$
}

Department of Pediatrics, Sporting Student Hospital [Alexandria], Egypt ${ }^{[a]}$

Department of Pediatrics, Damietta Faculty of Medicine, Al-Azhar University, Egypt ${ }^{[b]}$

Department of Pediatrics, Faculty of Medicine, Alexandria University, Egypt[c]

Corresponding author: Nada Alaa Mohamed Diab

Email: nadadiab844@gmail.com

Submitted at: February 24, 2020; Revised at: April 15, 2020; Accepted at: April 15, 2020; Available online at: April 15, 2020

DOI: 10.21608/ijma.2020.24557.1106

\section{ABSTRACT}

Background: Mitral valve prolapse is common with a reported prevalence ranging from 2-3\%, Mitral valve prolapse and its possible complications is not yet studied in Egyptian children.

Aim of the work: To study the frequency of mitral valve, prolapse in apparently healthy children aging between 3 to 15 years old.

Patients and Methods: A cross sectional study, the study sample contained 150 apparently healthy child selected by nonsystemic random sample, 78 males (52.0\%) and 72 females (48.0\%), with age ranged from 3-15 years old, the mean age was 2.36 years, living at Alexandria governorate and fulfilling the inclusion criteria coming for outpatient office visits for well child care at sporting students' hospital, Alexandria, through the period from March 2018 through April 2019.

Results: The frequency of mitral valve prolapse in children in Alexandria governorate was found in 18 cases (12.0\%), 17 cases of them (11.4\%) had mitral regurge [MR]. MVP was more frequent among females $(63.6 \%)$ than males $(36.4 \%)$ but the difference did not reach statistically significant difference $(p>0.05)$. echocardiographic examination reflects the mild nature of the disease.

Conclusion: Mitral valve prolapse reported in $12 \%$ of apparently healthy children, which reflects the magnitude of the problem. Female children were more affected. This indicates the importance of screening healthy children to early diagnose the condition and permit early treatment.

Keywords: Echocardiography; Mitral; Prolapse; Regurgitation; Prevalence

This is an open access article under the Creative Commons license [CC BY] [https://creativecommons.org/licenses/by/2.0/]

Please cite this article as: Diab NAM, El-Seheimy LA, Abd Elmohsen AM. Frequency of Mitral Valve Prolapse in Apparently Healthy Children in Alexandria Governorate. IJMA 2020; 2[2]: 462-468.

* Main subject and any subcategories have been classified according to researchers' main field of study. 


\section{INTRODUCTION}

Mitral valve prolapse is a valvular heart disease characterized by an abnormally thickened mitral valve leaflet being displaced into the left atrium during systole. Prolapsed mitral valves are divided into several subtypes depending on the thickness of the leaflet, concavity and degree of contact to the annulus of the mitral. Subtypes can be defined as classical, non-classical, symmetrical, asymmetrical, flail or non-flail. MVP bears a low risk of complications in its non-classical form [1],

Prolapse of the mitral valve is frequent with a reported prevalence of $2-3 \%$. Many will continue to develop extreme mitral regurgitation (MR) that would require intervention. The rate of disease progression, however, remains elusive. MVP pathophysiology is thought to be a continuing pattern of frequent minor injury and repair arising in a mitral valve with small congenital anatomical differences in the valve apparatus during the cardiac cycle. This may explain the production and progression of prolapse based on age [2]. This identifies two distinct types of "mitral valve prolapse (MVP)". Also known as mitral valve prolapse syndrome (MVPS), is the primary or classic form. It is associated with mitral valve leaflet defects, as well as perivalvular supporting tissue such as chordae tendineae and annulus. MVPS is also associated with additional cardiac symptoms such as skeletal, connective tissue, and characteristics of neuropsychiatry. The defect in the secondary or nonclassical form is confined to the mitral valve without perivalvular or systemic manifestations [3].

The most useful method for diagnosis of a prolapsed mitral valve is echocardiography. Twodimensional echocardiography and threedimensional echocardiography are particularly valuable because they allow visualization of mitral leaflets relative to an annulus. This allows measurement of the thickness relative to the annulus and their displacement. Thickening of mitral leaflets $>5 \mathrm{~mm}$ and displacement of leaflets $>2 \mathrm{~mm}$ indicates a typical prolapse of the mitral valve ${ }^{[4]}$. Echocardiography remains probably the most appropriate diagnostic form for mitral prolapse. Computed tomography is limited by the need for ionizing radiation and the failure to diagnose mitral prolapse accurately [5,6].
Cardiac magnetic resonance imaging is improving and can reliably measure the degree of MR; however, leaflet thickening is difficult to assess given current spatial resolution constraints $[7]$.

MVP development may occur in stages over a lifetime, beginning with a genetic substratum, progressing to mild, non-diagnostic valve morphologies, developing into a full expression of the MVP phenotype, and culminating in serious MR that needs valve surgery. The period of the individual stages of the disease can vary from months to years, the shorter duration of the progression being the result of a flail mitral leaflet. The presence of early MVP morphologies in the general population would provide an incentive for echocardiographic screening and possible intervention at an early stage of the disease ${ }^{[8]}$.

Mitral valve prolapse and its possible complications are not yet studied in Egyptian children, thus, it makes me enthusiastic to study its prevalence in the children who are attending the outpatient clinic of Sporting Health Insurance Students Hospital.

\section{AIM OF THE WORK}

This work aimed to study the frequency of mitral valve prolapse in apparently healthy children aging between 3 to 15 years old.

\section{PATIENTS AND METHODS}

After obtaining approval of the ethics committee, a cross-sectional study was carried out on 150 children living at Alexandria governorate and fulfilling the inclusion criteria coming for outpatient office visits for well child care at sporting students' hospital, Alexandria, through the period from March 2018 through April 2019. The sample size was calculated using Epi Info7.2. In a previous study, the estimated prevalence of mitral valve prolapse was 0.13 . If the total number of children attending the clinic (from March 2018 through April 2019.) was 720 (average 20 outpatient visits per day), we will need to study 140 children to estimate the prevalence with $95 \%$ confidence level, $80 \%$ power and type I error probability of 0.05 . The protocol was accepted by the Faculty of Medicine's ethical review committee at Al Azhar University. The work was carried out for human studies following the World Medical Association's Code of Ethics (Helsinki Declaration). 
Inclusion criteria: Age: between 3 to 15 years old, sex: both sexes. Exclusion criteria: Patients with known cardiac disease. Patients with a prolapse of the mitral valve by tapping or murmuring.

Data collection: All children of the study were subjected to Complete history taking includes: Age, sex and residency of the patient, duration of illness, and associated cardiovascular symptoms. Thorough clinical evaluation with special emphasis on local cardiac examination. Full echocardiographic study including two dimensional and Doppler studies.

Echocardiographic Methods: All participants were subjected to standard two-dimensional echocardiography with a commercially available device (Sonos 1000, Hewlett - Packard, Andover, Mass). Images were captured on videotape including complete parasternal, apical, and subcostal views and valvular regurgitation color Doppler assessment. All tests were conducted with a Cardiac Analysis Device Sony Off-line (Sum 1010, Sony, Park Ridge, $\mathrm{NJ})$. Based on the three-dimensional structure of the annulus and clinical similarities, we used existing two-dimensional echocardiographic parameters to diagnose mitral-valve prolapse according to the average superior displacement of the mitral leaflets during the systole relative to the line connecting the annular-hinge dots. In the parasternal and apical long-axis views we measured the displacement of the anterior and posterior mitral leaflets, which were tested by tilting the transducer to image the three scallops of the posterior leaflet.

Statistical Analysis: Using IBM SPSS software package version 20.0, the data was fed to the personal computer. Using numbers and percentages, qualitative data were represented. Chisquare test was used to evaluate associations of categorical variables between different groups. Quantitative data have been defined using mean and standard deviation for normally distributed data while abnormally distributed data has been represented using median, minimum and maximum. For normally distributed data, a comparison was made using $t$ tests between two independent populations, while Ftest (ANOVA) was used with more than two populations. The results of the significance test are stated as two-tailed probabilities. The significance of the results obtained was calculated at the point of $5 \%$.

\section{RESULTS}

Table (1) showed the distribution of the studied group regarding their age and sex, it was found that the mean age of the study group was $8.20 \pm 2.36$, the majority of the studied group was in age group 6-9 years, the male represent $52.0 \%$ of the studied group and the female was $48.0 \%$. Table (2) showed the distribution of the studied patients' group regarding MVP, the majority of the studied group (88.0\%) showed normal 2D echocardiographic findings, 17 cases $(11.3 \%)$ had a mild MVP, and only one case $(0.7 \%)$ had moderate MVP. Table (3) demonstrated the relation between MVP findings and associated cardiovascular symptoms, it was found that there was no significant relation between associated cardiovascular symptoms and MVP findings ( $p$ $>0.01$ ). Table (4) revealed the relation between cardiac auscultatory findings and MVP, there was no significant relation, $(p>0.05)$.

Figure (1) showed the correlation between MR findings and MVP, it was found that there was a significant relation between MR findings by $2 \mathrm{D}$ and MVP by Doppler as there was 10 of 11 with MVP had $\operatorname{MR}(p<0.05)$.

Table [1]: Distribution of the studied group regarding their age and sex

\begin{tabular}{|c|c|}
\hline & Statistics \\
\hline $\begin{array}{c}\text { Age group (years) }(\mathrm{n}, \%) \\
<6 \text { years } \\
6-9 \\
>9\end{array}$ & $\begin{array}{l}21(14.0 \%) \\
77(51.3 \%) \\
52(34.7 \%)\end{array}$ \\
\hline $\begin{array}{l}\text { Range } \\
\text { Mean } \\
\text { S.D. } \\
\text { Median }\end{array}$ & $\begin{array}{l}4-15 \\
8.20 \\
2.36 \\
8.00\end{array}$ \\
\hline Sex & $\begin{array}{l}78(52.0 \%) \\
72(48.0 \%)\end{array}$ \\
\hline
\end{tabular}


Table (2): Distribution of the studied group regarding MVP echocardiographic findings

\begin{tabular}{|l|c|}
\hline \multicolumn{1}{|c|}{ MVP echocardiographic findings } & Statistics \\
\hline Normal & $132(88.0 \%)$ \\
MVP & $18(12.0 \%)$ \\
Mild MVP & $17(11.3 \%)$ \\
Moderate MVP & $1(0.7 \%)$ \\
\hline
\end{tabular}

Table (3): Relation between MVP findings and cardiovascular symptoms.

\begin{tabular}{|l|c|c|c|c|}
\hline \multirow{2}{*}{ Cardiovascular symptoms } & \multicolumn{3}{|c|}{ MVP } & \multirow{2}{*}{ Total $(\mathrm{n}=150)$} \\
\cline { 2 - 5 } & None $(\mathrm{n}=132)$ & Mild $(\mathrm{n}=17)$ & Moderate $(\mathrm{n}=1)$ & \\
\hline No & $101(76.52 \%)$ & $11(64.71 \%)$ & $1(100.0 \%)$ & $113(75.3 \%)$ \\
\hline Vague chest pain & $12(9.09 \%)$ & $3(17.65 \%)$ & $0(0.0 \%)$ & $15(10.0 \%)$ \\
\hline Palpitations & $19(14.39 \%)$ & $3(17.65 \%)$ & $0(0.0 \%)$ & $22(14.67 \%)$ \\
\hline \multicolumn{3}{|c|}{$2.15 ; 0.21$} \\
\hline
\end{tabular}

Table (4): Relation between Cardiac auscultatory findings and MVP.

\begin{tabular}{|c|c|c|c|c|}
\hline \multirow[t]{2}{*}{ Cardiac auscultatory findings } & \multicolumn{3}{|c|}{ MVP (2D) } & \multirow[t]{2}{*}{ Total } \\
\hline & None $(n=132)$ & Mild $(n=17)$ & Moderate $(n=1)$ & \\
\hline Normal & $131(99.24 \%)$ & $15(88.24 \%)$ & $1(100.0 \%)$ & $147(98.0 \%)$ \\
\hline Ejection systolic murmur & $1(0.76 \%)$ & $2(11.76 \%)$ & $0(0.0 \%)$ & $3(2.0 \%)$ \\
\hline$X^{2} ; p$ & & & & \\
\hline
\end{tabular}

normal $\quad$ Trivial MR $\quad$ Mild MR $\quad$ Moderate MR

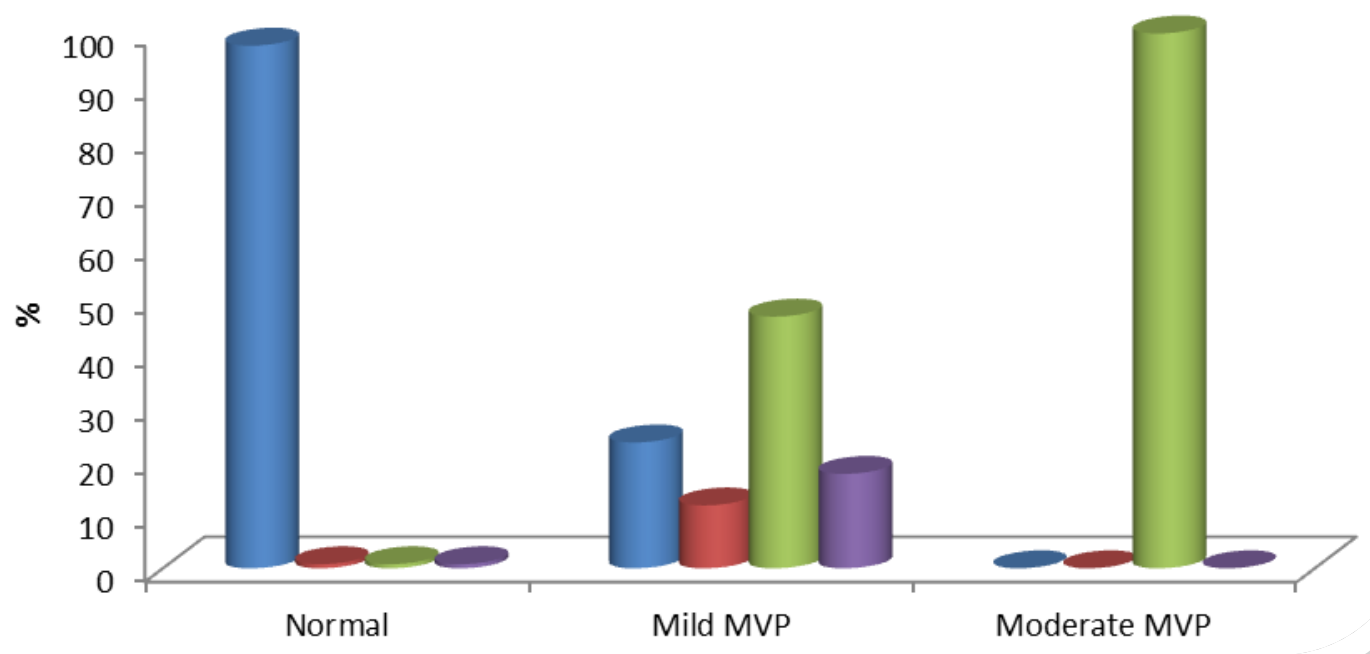

Figure (1): Association between MR findings and MVP

\section{DISUCSSION}

Cardiac whispering is widely reported in asymptomatic children and is the most common reason for referral to a cardiologist. The reported prevalence of cardiac murmurs in apparently healthy children varies widely, depending on the community examined, from 5 percent to 80 percent of the screened population ${ }^{[9]}$. Among infants and children, the majority of heart murmurs are regular or innocent. Many cardiac murmur prevalence reports come from early studies, pre-dating echocardiography and interventional cardiology, which have enhanced the accuracy of structural heart disease diagnosis (SHD)[?]. 
Numerous studies have established clinical predictors of pathological murmurings, but the lack of abnormal findings does not always guarantee the exclusion of SHD diagnosis. Echocardiography remains the formal treatment method in gold. [10].

Echocardiography is a very specific tool for the diagnosis and characterization of SHD. The advent of high-quality, portable echocardiography systems means that the cause of murmurings in school-aged children can be confirmed on-site [10].

In our results, it was found that 3 children $(2.0 \%)$ had ejection murmur. In agreement with our study, other paper carried out by Kang et al., [9], they found that $2193(2.7 \%)$ of 81213 who underwent cardiac examination, were found to have a murmur. All the schoolchildren who had a murmur were subjected to echocardiography, confirming an SHD in 215. The murmurs ascertained in 1978 were classified as innocent murmurings. Of the students who had an SHD, 198 had a CHD, 12 had an MVP and 5 had an $\mathrm{RHD}$. As for age, SHDs were distributed equally among males and females[9].

In the previous study, there was a normal echocardiographic examination of about 9 out of 10 schoolchildren who were referred for a murmur evaluation. In our research, which was also consistent with many other studies, we found the decreasing prevalence of $\mathrm{CHD}$ with increasing age. This means we can reduce the number of unneeded echocardiographic exams in children over 10 years of age [9].

Regarding MVP in our study, the majority of the studied group (88\%) show normal 2D echocardiographic findings, the overall prevalence was 18 cases (12.0\%), 17 cases (11.3\%) had a mild MVP, only one case $(0.7 \%)$ had moderate MVP.

In agreement with our study, in Guy et al.[11], Study found that echocardiographic patterns of mitral valve closure in 193 normal children, superior systolic movement of at least one mitral valve leaflet above the mitral annular plane was found to be present in $13 \%$ of this population. The emergence of mitral valve prolapse in almost $15 \%$ of a group of normal children treated for exclusion indicates the need to redefine [11].

Like our research, Sattur et al. studied the prevalence of mitral valve prolapse and related valve regurgitations in healthy adolescents undergoing screening echocardiography, they found the prevalence to be below $10.0 \%$ [12].

In our study, it was found that MVP was more frequent among females $(63.6 \%)$ than males $(36.4 \%)$ but this was not a statistically significant difference $(p>0.05)$.

This result was agreement with Delling et al.[13] who found that the prevalence of MVP was similar in the two sexes, females $56.0 \%$ and male $44.0 \%$; In the FHS (Framingham Cardiac Study) Freed et al.[14], a free, community-based sample of referrals.

In contrast, in the Olmsted County population, characterized by a mixed spectrum of communitydwelling and referred patients, women were diagnosed with MVP more often than men and at a younger age using similar echocardiographic criteria[2].

Regarding cardiovascular symptoms in this study, it was found that there was no significant relation between associated cardiovascular symptoms and MVP findings ( $p>0.01)$. In agreement with our study, in a study carried out by Avierinos et al., ${ }^{14]}$, they found that absence of symptoms of MVP in positive cases, It remains unclear whether MVP is directly linked to autonomic dysfunction or is purely incidental to any recorded association[15].

In contrast to our results, Shah et al. found The MVP was associated with various symptoms (including atypical chest pain, exertional dyspnea, palpitations, syncope, and anxiety) and clinical findings (low blood pressure, leaner build-up, and electrocardiographic repolarization abnormalities) and their constellation was called MVP syndrome[ ${ }^{[16]}$.

Prolapse of the mitral valve causes no symptoms, and is often blamed for symptoms such as chest pain, palpitations, and lightheadedness. Individuals with mitral valve prolapse may have these symptoms, but the prolapse of the mitral valve is not usually the cause ${ }^{[17]}$.

In our study, the relation between Cardiac auscultatory findings and MVP showed insignificant relation ( $p>0.05$ ), the ejection systolic murmur was found in 3 cases, two of them was normal MVP and only on case showed mild MVP. This result was agreement with the study carried out by Delling et al.[13], they found that the relation between murmur and auscultation was insignificant $(p>0.05)$ the 2 
classes (with and without parental MVP) had similarly small numbers of persons with auscultation murmur (diastolic or systolic ejection), pointing out that the prevalence of clinically observable valve disease from all causes was weak and could not properly distinguish persons with family MVP.

In our results, when we study the correlation between MR findings and MVP, it was found that there was a significant relation between MR findings and MVP as there was 17 of 18 with MVP had MR ( $p$ $<0.05)$. In agreement with our study, Rajani et al., [18], study the Mitral valve prolapse and incidence of MR, they found that the mitral regurgitation show a significant association with MVP. Another study by Turker et al., on 58 children with mitral valve prolapse showed that the incidence of MR is increased especially accompanied by Moderate to severe regurgitation [19].

The relationship between MR and mitral valve prolapse has been studied to a certain extent. Bobkowski et al. evaluated 113 children with mitral valve prolapse against 101 healthy children and concluded that MR was significantly higher in the mitral valve prolapse group[20]. Similar results were reported by Digeos-Hasnier et al. ${ }^{21]}$.

In Conclusion, for several reasons it is important to assess the prevalence of mitral valve prolapse in the general population. It determines the severity of the condition and acts as a basis for determining the validity of the associations proposed. It also allows researchers to examine whether prolapse occurs more often in patients with suspected complications, such as stroke, than in the general population.

Prospective studies are necessary to identify which child with MVP is gradually developing MVP, and will have potential clinical symptoms. Long-term follow-up and further prospective studies of these children with MVP are needed to find out about MVP's natural history. Follow-up of these individuals can show whether the existence of MVP is an early indicator of subclinical autoimmunity and may lead to the early diagnosis and therapeutic prevention of an overt autoimmune disease, as well as the potentially serious complications of MVP.

\section{Financial and Non-Financial Relationships and Activities of Interest}

None

\section{REFERENCES}

1. Hayek E, Gring CN, Griffin BP. Mitral valve prolapse. Lancet 2005; 5-11365 (9458): 507-18. [DOI: 10.1016/ S0140-6736(05) 17869-6].

2. Delling FN, Rong J, Larson MG, Lehman B, Fuller D, Osypiuk E, Levine RA. Evolution of mitral valve prolapse: insights from the Framingham heart study. Circulation 2016; 133:1688-95. [DOI: 10.1161/ CIRCULATIONAHA. 115. 020621]

3. Levine HJ, Isner JM, Salem DN. Primary versus secondary mitral valve prolapse: clinical features and implications. Clin Cardiol. 1982; 5:371-5. [DOI: 10.1002/ clc. 4960050605].

4. Playford D, Weyman AE. Mitral valve prolapse: time for a fresh look. Rev Cardiovasc Med. 2001; 2(2):73-81. [DOI: 10.1016/s0008-6363(00) 00251-0].

5. Smith T, Gurudevan S, Cheng V, Trento A, DeRobertis M, Thomson L, Berman DS. Assessment of the morphological features of degenerative mitral valve disease using 64-slice multidetector computed tomography. J Cardiovasc Comput Tomogr. 2012; 6:415-21. [DOI: 10. 1016/j.jcct. 2012.10.004].

6. Ghosh N, Al-Shehri H, Chan K, Mesana T, Chan V, Chen L, Chow B J. Characterization of mitral valve prolapse with cardiac computed tomography: comparison to echocardiographic and intraoperative findings. Int $\mathrm{J}$ Cardiovasc Imaging. 2012; 28:855-63. [DOI: 10.1007/ s10554- 0119888-0].

7. Chan KM, Wage R, Symmonds K, Rahman-Haley S, Mohiaddin RH, Firmin DN, Kilner PJ. Towards comprehensive assessment of mitral regurgitation using cardiovascular magnetic resonance. J Cardiovasc Magn Reson 2008; 10:61. [DOI: 10.1186/1532-429X-10-61].

8. Nesta F, Leyne M, Yosefy C, Simpson C, Dai D, Marshall $\mathrm{JE}$, et al. A new locus for autosomal dominant mitral valve prolapse on chromosome 13: clinical insights from genetic studies. Circulation. 2005; 112: 2022-2030. [DOI: 10.1161/ CIRCULATIONHA. 104.516930].

9. Kang G, Xiao J, Wang Y, Wang J, Chen Y, Liu Q, et al. Prevalence and clinical significance of cardiac murmurs in schoolchildren. Archives of disease in childhood. 2015; 100 (11): 1028-1031. [DOI: 10.1136/archdischild-2014-307819].

10. Brown JL, Hirsh DA, Mahle WT. Use of troponin as a screen for chest pain in the pediatric emergency department. Pediatr Cardiol. 2012; 33(2): 337-42. [DOI: 10.1007/ s00246-011-0149-8].

11. Guy TS, Hill AC. Mitral valve prolapse. Annu Rev Med 2012; 63:277-92. [DOI: 10.1146/annurev-med-022811091602].

12. Sattur S, Bates S, Movahed MR. Prevalence of mitral valve prolapse and associated valvular regurgitations in 
healthy teenagers undergoing screening echocardiography. Exp Clin Cardiol. 2010; 15(1): e13-e15. [DOl: 1186/1476-7120-8-54].

13. Delling FN, Rong J, Larson MG, Lehman B, Osypiuk E, Stantchev P, et al. Familial clustering of mitral valve prolapse in the community. Circulation, 2015; 131(3): 2635. [DOI: 10.1161/ CIRCULATIONAHA.114.012594].

14. Freed LA, Levy D, Levine RA, Larson MG, Evans JC, Fuller DL, Lehman B, Benjamin EJ. Prevalence and clinical outcome of mitral-valve prolapse. $\mathrm{N}$ Engl J Med. 1999; 341:1-7. [DOI: 10.1056/NEJM 19990701341 0101].

15. Avierinos JF, Detaint D, Messika-Zeitoun D, Mohty D, Enriquez-Sarano M. Risk, determinants, and outcome implications of progression of mitral regurgitation after diagnosis of mitral valve prolapse in a single community. Am J Cardiol. 2008; 101:662-667. [DOI: 10.1016/j. amjcard.2007.10.029].

16. Shah PM. Current concepts in mitral valve prolapsediagnosis and management. J Cardiol. 2010; 56:125-133. [DOI: 10.1016/j.jjcc.2010.06. 004].

17. Bonow RO, Carabello B A, Chatterjee K, de Leon Jr AC, Faxon DP, Freed MD, O'Rourke RA. American College of Cardiology/ American Heart Association Task Force. 2008 focused update incorporated into the ACC/AHA 2006 guidelines for the management of patients with valvular heart disease. Circulation, 2008; 118(15): e523-661. [DOI: 10.1161/CIRCULATIONAHA.106.177303].
18. Rajani A, Murugesan V. Baslaib F, Rafiq M. Mitral valve prolapse and electrolyte abnormality: a dangerous combination for ventricular arrhythmias. BMJ Case Rep 2014; bcr 2014205055 [DOI: 10.1136/bcr-2014-205055].

19. Turker Y, Ozaydin M, Acar G, Ozgul M, Hoscan Y, Varol E, Yucel H. Predictors of ventricular arrhythmias in patients with mitral valve prolapse. Int J Cardiovasc Imaging 2010; 26:139-45. [DOI: 10.1007/s10554-009-9514-6].

20. Bobkowski W, Nowak A, Durlach J. The importance of magnesium status in the pathophysiology of mitral valve prolapse. Magnes Res 2005; 18: 35-52. [DOI: 10.1142/97898127022 34_0199]

21. Digeos-Hasnier S, Copie X, Paziaud O, Abergel E, Guize L, Diebold B, Le Heuzey JY. Abnormalities of ventricular repolarization in mitral valve prolapse. Ann Noninvasive Electrocardiol 2015; 10:297-304. [DOI: 10.1111/j.1542474X. 2005.00630.x]. 